\title{
A qualitative analytical investigation of geometrically nonlinear effects in wind turbine blade cross sections
}

\author{
Eder, Martin Alexander; Bitsche, Robert
}

Published in:

Thin-Walled Structures

Link to article, DOI:

10.1016/j.tws.2015.03.007

Publication date:

2015

Document Version

Early version, also known as pre-print

Link back to DTU Orbit

Citation $(A P A)$ :

Eder, M. A., \& Bitsche, R. (2015). A qualitative analytical investigation of geometrically nonlinear effects in wind turbine blade cross sections. Thin-Walled Structures, 93, 1-9. https://doi.org/10.1016/j.tws.2015.03.007

\section{General rights}

Copyright and moral rights for the publications made accessible in the public portal are retained by the authors and/or other copyright owners and it is a condition of accessing publications that users recognise and abide by the legal requirements associated with these rights.

- Users may download and print one copy of any publication from the public portal for the purpose of private study or research.

- You may not further distribute the material or use it for any profit-making activity or commercial gain

- You may freely distribute the URL identifying the publication in the public portal 


\title{
A qualitative analytical investigation of geometrically nonlinear effects in wind turbine blade cross sections
}

\author{
M.A. Eder ${ }^{\mathrm{a}}$, R.D. Bitsche ${ }^{\mathrm{b}}$ \\ ${ }^{a}$ Corresponding author: maed@dtu.dk, Technical University of Denmark, Frederiksborgvej \\ 399, 4000 Roskilde, Denmark \\ ${ }^{b}$ robi@dtu.dk, Technical University of Denmark, Frederiksborgvej 399, 4000 Roskilde, \\ Denmark
}

\begin{abstract}
This article analytically investigates the Brazier effect on asymmetric thinwalled sections subject to biaxial bending. In the latter case a torsional moment - in this paper referred to as Brazier torsion - is induced, which proved to be a vital part of the solution. By means of a generic cross section, that was inspired by a wind turbine blade it is demonstrated that geometric nonlinear effects can induce an in-plane opening deformation in re-entrant corners that may decrease the fatigue life. The opening effect induces Mode-I stress intensity factors which exceed the threshold for fatigue crack growth at loads well below the load-carrying capacity of the beam.

The findings in this paper are twofold: Firstly, the investigated analysis procedure can be integrated into the design process of wind turbine blade cross sections. Secondly, the proposed approach serves as a basis for computationally efficient numerical analysis approaches of structures that comprise complex geometry and anisotropic material behaviour - such as wind turbine rotor blades.
\end{abstract}

Keywords:

Geometrically nonlinear effects, Brazier effect, Wind turbine blade, Trailing edge, Fatigue threshold

\section{Introduction}

The investigation of tubes under large pure bending deformation was initiated by von Kármán [1] for curved circular tubes, and for rectangular hollow sections by Timoshenko [2], and was later modified for initially straight tubes 
by Brazier [3]. The well-known Brazier effect deals with the cross sectional capacity limit upon second-order in-plane deformations that lead to ovalisation of circular sections (Fig. 1 (a)). The ovalisation is caused by in-plane deviation stresses as shown in Fig.1 (b). The Brazier effect has been studied extensively by many researchers, such as Clark and Reissner [4] and Reissner [5] to name only a few. Recently Guarracino [6] investigated the formation of axial wrinkles at the compressed region where Corona and Rodrigues [7] investigated bifurcation buckling of orthotropic circular tubes. This shows that research on the Brazier effect focussed earlier on the second-order capacity limits of tubular cross sections, but more recently on the transition of the Brazier effect into local stability limits (i.e. local buckling). Moreover, Guarracino et al. [8], [9] and [10] experimentally, numerically and analytically investigated local effects imposed by collars and supports as they e.g. appear in pipelines. They pointed out that counterintuitive implications of the Brazier effect can cause significant deviations of axial tensile and compressive strains obtained from simple bending theory.

Although the Brazier effect is present in all cross sections it becomes mostly apparent in thin-walled hollow sections. A typical representative of such a thin-walled multi-cellular cross section is that of a wind turbine blade as depicted in Fig.1 (c).

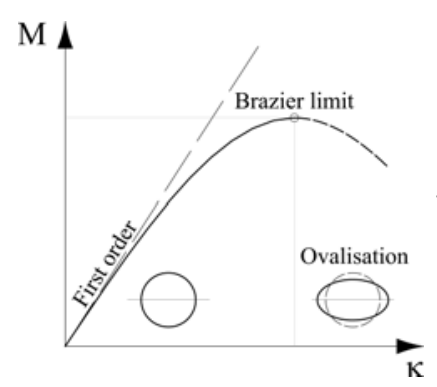

(a)

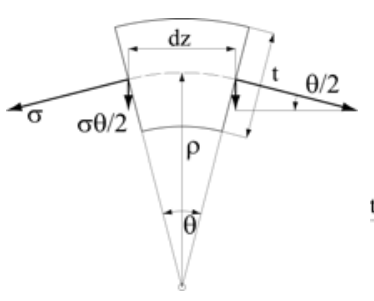

(b)

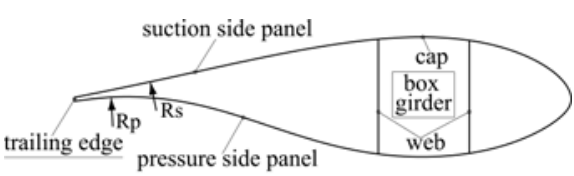

(c)

Figure 1: (a) Bending moment curvature responses for first-order (dashed line) and secondorder (solid line) cross-section capacity due to ovalisation; (b) Infinitesimal element subject to bending stress $\sigma$ and second order in-plane deviation stress responsible for Brazier effect; (c) Sketch of a typical wind turbine rotor blade cross section; the aerodynamic shell and the box girder form a tri-cellular cross section; pressure side panel and suction side panel are joined at a re-entrant corner which is referred to as trailing edge. Both panels feature a curvature whose local radii are denoted as $R_{p}$ and $R_{s}$, both of which have a significant influence on the aerodynamic performance of the airfoil. 
Wind turbine blades are cantilever glass fibre reinforced polymer composite beams that can undergo considerable bending deformations of up to $20 \%$ of their span. In the design of wind turbine blades the classic Brazier limit is usually not relevant. Kühlmeier [11] showed that the local buckling limit of wind turbine blades is usually lower than the Brazier limit moment. Nevertheless, Damkilde and Lund [12] showed that the Brazier effect induces transverse stresses in the cap of the main carrying box girder of wind turbine blades. Due to the distinct orthotropic material behaviour of caps, it was shown that these transverse stresses can reach the transverse tensile strength of the material at load levels below the Brazier limit. This finding was significant as the structural impact of the Brazier effect can no longer be neglected in the blade design process.

Recent experimental and numerical investigations of fracture mode deformations near the trailing edge of a wind turbine blade [13] showed that in-plane deformations could be attributed to the Brazier effect. More importantly it could be seen that the trailing edge 'opened' for certain loading directions and load levels far below the capacity limit of the blade, all of which makes the trailing edge susceptible to fatigue damage.

It was Cecchini and Weaver [14] who initially shed light on geometric nonlinear effects affecting the trailing edge. They provided an analytical approach for the Brazier effect in a simplified symmetric airfoil that is subjected to pure flapwise bending. Following the concept of Brazier, they reduced the $3 \mathrm{D}$ problem to a $2 \mathrm{D}$ problem which can be solved both analytically and numerically on a cross-sectional level. Cecchini and Weaver subsequently proposed a finite-element-based approach in which fully non-linear deformations can be efficiently obtained from a thin cross sectional slice. They eventually showed - by means of a NACA0012 profile [15] - that flapwise bending (bending around the minor principal axis) leads to a closing of the trailing edge. This behaviour can be intuitively explained by the symmetrically distributed Brazier pressure that consequently squeezes the trailing edge panels together.

However, the trailing edge deformation behaviour becomes less intuitive for asymmetric, curved, thin-walled sections subject to bending about other than the principal axes. The analysis of such a general case is not straightforward owing to a torsional moment that is induced into the deformed cross section which must be considered in order to satisfy the equilibrium conditions. It will be shown in this paper that certain asymmetric, curved thin- 
walled sections exhibit a counterintuitive opening effect of the trailing edge under certain bending directions which is consistent with both experimental and numerical analyses.

The aim of this paper is to analytically investigate the source of this effect in order to aid the future development of structurally improved airfoil geometries. Owing to the complexity of wind turbine blades with regard to geometry and anisotropic material behaviour this effect will be demonstrated on a simplified example. Moreover, it must be stressed that nonlinear numerical fracture analyses of 3D wind turbine rotor blade models are computationally expensive. This paper follows the modelling approach of [14] and aims to facilitate a more efficient numerical analysis approach of asymmetric airfoil slices for bi-axial bending directions.

\section{Methods}

\subsection{Brazier torsion}

The Brazier pressure acting at a point on the centre line of the isotropic, linear elastic, thin-walled section shown in Fig. 1 (b) can be written with $\theta=\mathrm{d} z / \rho=\kappa \mathrm{d} z$ as

$$
\mathrm{d} p=\sigma t \theta=\kappa^{2} E t r \mathrm{~d} z
$$

where $\theta$ represents the angle of bending rotation, $\rho$ denotes the bending radius, $\kappa$ represents the St.Venant bending curvature, $E$ denotes the elastic modulus, $t$ denotes the wall thickness, $r$ represents the distance from that point perpendicular to the bending axis and $\mathrm{d} z$ represents the thickness of an infinitesimal cross sectional slice of the main beam. The Brazier pressure has the unit force per circumferential wall length $\mathrm{d} l$. Clearly, the Brazier pressure vector always points perpendicularly towards the bending axis (i.e. axis of curvature). With the position vector $\vec{r}=\{x, y\}^{T}$ and using the transformation matrix

$$
T=\left[\begin{array}{cc}
\cos \alpha & -\sin \alpha \\
\sin \alpha & \cos \alpha
\end{array}\right]
$$

the pressure Brazier vector can be written as

$$
\mathrm{d} \vec{p}=-\kappa^{2} E t \mathrm{~d} z\left\{\begin{array}{l}
\sin \alpha^{2} x+\sin \alpha \cos \alpha y \\
\sin \alpha \cos \alpha x+\cos \alpha^{2} y
\end{array}\right\}
$$

where $x$ and $y$ are the Cartesian coordinates of an arbitrary point on the wall

with its origin in the elastic centre $C_{E}$ and $\alpha$ represents the angle between 
the bending axis (i.e. axis of cross section rotation) and the positive $\mathrm{x}$-axis as shown in Fig.2 (a).
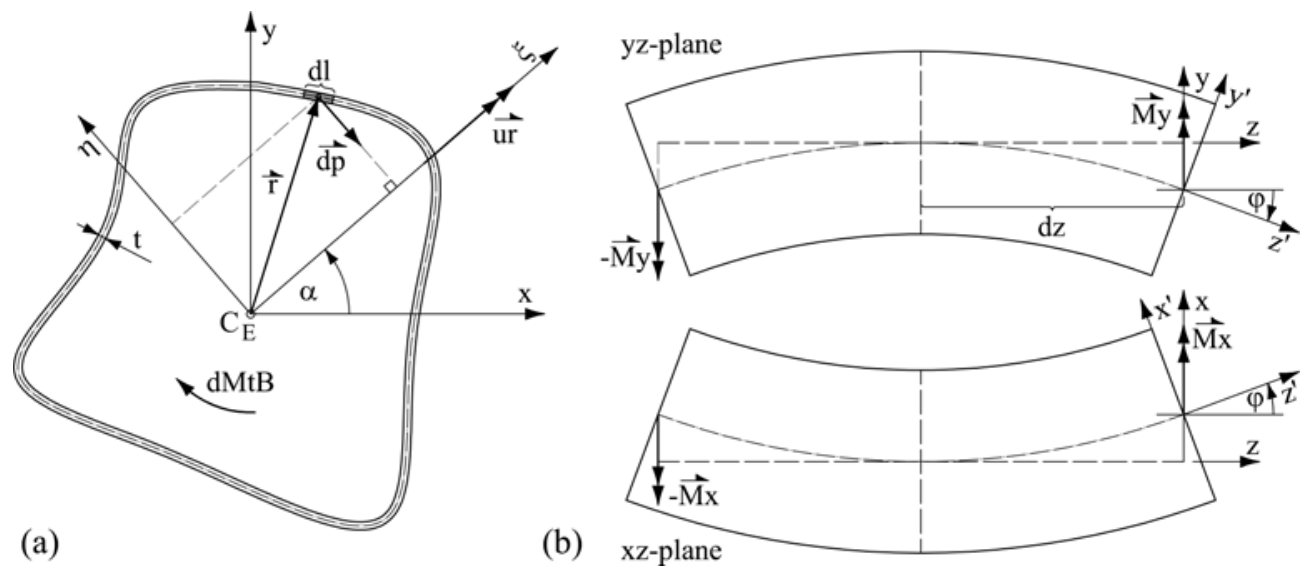

Figure 2: (a) Arbitrary thin-walled tubular cross section subjected to biaxial bending denoted by rotation vector $\overrightarrow{u r}$. Principal cross section coordinate system $x, y$ and the bending coordinate system aligned with bending axis $\xi, \eta$; (b) Deformed main beam element (Euler-Bernoulli hypothesis) subjected to biaxial bending $\vec{M}_{x}, \vec{M}_{y}$ depicted in two orthogonal views with the coordinate system of the deformed cross section $x^{\prime}, y^{\prime}$

The Brazier pressure given in equation 3 induces a torsional moment per unit wall length around the elastic centre which can be written as the crossproduct $\mathrm{d} M t_{B}=\mathrm{d} \vec{p} \times \vec{r}=\kappa^{2} E t \mathrm{~d} z\left(\sin \alpha \cos \alpha\left(x^{2}-y^{2}\right)+\left(2 \cos \alpha^{2}-1\right) x y\right)$. Integration over the cross section with $\mathrm{d} A=t \mathrm{~d} l$ yields the total torsional moment in this paper referred to as Brazier torsion

$$
\begin{aligned}
M t_{B} & =\int \mathrm{d} M t_{B} \mathrm{~d} l=\kappa^{2} E \mathrm{~d} z\left(\sin \alpha \cos \alpha\left(I_{y}-I_{x}\right)+\left(2 \cos \alpha^{2}-1\right) I_{x y}\right) \\
& =\kappa^{2} E t \mathrm{~d} z \int \xi \eta \mathrm{d} l=\kappa^{2} E \mathrm{~d} z I_{\xi \eta}
\end{aligned}
$$

where $I_{x}$ and $I_{y}$ represent both second moments of area and $I_{x y}$ represents the deviatoric moment of area in the principal coordinate system (CSYS) and $I_{\xi \eta}=[T]^{-1}\left[\begin{array}{cc}I_{x} & I_{x y} \\ I_{x y} & I_{y}\end{array}\right][T]$ represents the deviatoric moment of area in a CSYS aligned with the bending axis.

It can easily be seen from Fig.2 (a) that the infinitesimal Brazier torsion $\mathrm{d} M t_{B}$ is caused by the Brazier pressure times its perpendicular distance to 
the elastic centre. Equation 4 shows that $M t_{B}$ is, therefore, a function of the deviatoric moment of area in the $\xi, \eta$ CSYS where the expression in the parenthesis of equation 4 is simply the transformation of $I_{\xi \eta}$ into the principal CSYS. The Brazier torsion is zero if $I_{\xi \eta}=0$ which applies to all 90-degree rotationally symmetric cross sections such as a square, irrespective of the angle $\alpha$.

A different view of the Brazier torsion can be obtained by considering the externally applied principal bending moments $M_{x}$ and $M_{y}$ in the deformed CSYS. Linearisation of the cross-sectional rotations $\varphi_{x}=\kappa_{x} \mathrm{~d} z=M_{x} \mathrm{~d} z / E I_{x}$ and $\varphi_{y}=\kappa_{y} \mathrm{~d} z=M_{y} \mathrm{~d} z / E I_{y}$ of an infinitesimally long main beam element $\mathrm{d} z$ as shown in Fig. 2 (b), lets the torsional moment be written as

$$
M t_{B}=M_{y} \varphi_{x}-M_{x} \varphi_{y}=\frac{M_{x} M_{y}}{E} \mathrm{~d} z\left(\frac{1}{I_{x}}-\frac{1}{I_{y}}\right)=\left(\begin{array}{c}
\kappa_{x} \\
\kappa_{y}
\end{array}\right) \cdot\left(\begin{array}{c}
M_{y} \\
-M_{x}
\end{array}\right) \mathrm{d} z
$$

It can be shown that substitution of $\kappa^{2}=1 / E^{2}\left(M_{y}^{2} / I_{y}^{2}+M_{x}^{2} / I_{x}^{2}\right)$ and $\sin \alpha \cos \alpha=M_{x} I_{y} M_{y} I_{x} /\left(M_{x}^{2} I_{y}^{2}+M_{y}^{2} I_{x}^{2}\right)$ into equation 4 indeed gives equation 5. In other words, a beam with $I_{\xi \eta} \neq 0$ exhibits a second-order coupling between bending and twisting as a matter of moment vector components being projected onto the $z^{\prime}$-axis.

\subsection{Circular hollow section}

The geometrically nonlinear deformations of a cross section can be obtained by considering the walls of the cross section as beams themselves loaded by externally applied Brazier pressure. These beams are referred to as cross-section beams in this paper as shown in Fig. 4. In this subsection it will be shown that $2 \mathrm{D}$ beam analysis of a circular initially straight tube yields the solution found by Brazier [3]. For this purpose the commercially available algebraic manipulation software Maple [16] was used. In the proposed approach the cross section is modelled as a circular cross-section beam with a width of $\mathrm{d} z$ and thickness $t$. The in-plane extensional stiffness is hence given by $E A=E t \mathrm{~d} z$ where the in-plane bending stiffness is given by $E I=E t^{3} \mathrm{~d} z / 12$. Using symmetry, only the upper semi-circular arc was considered where the left end was fully fixed and the right end was rotationally restrained but free to move in the x-direction as shown in Fig. 3 (a). Figure 3 (b) shows that two flexural hinges were introduced at both ends in order to make the system statically determined. The theory of slightly 

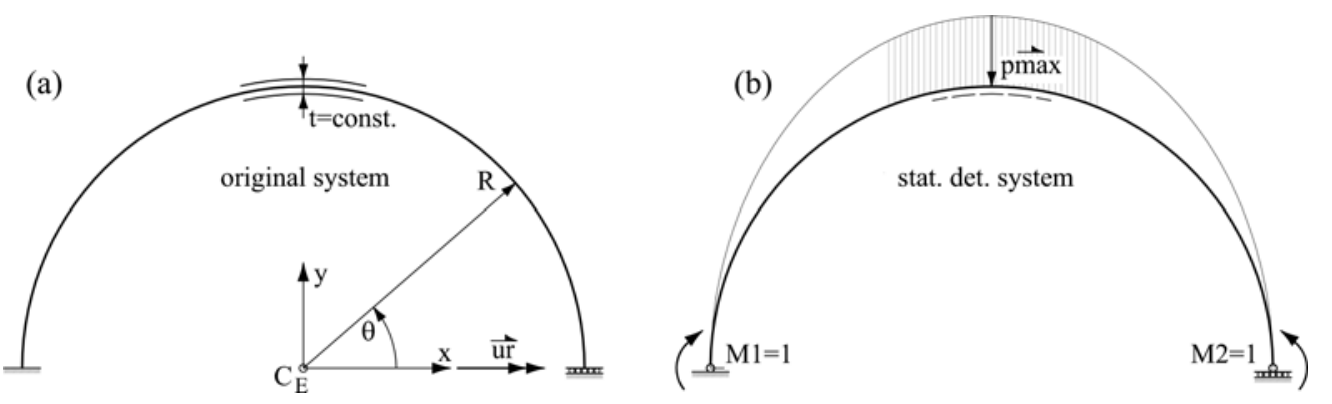

Figure 3: (a) Half of the original system of a circular cross section with constant wall thickness. Curvature was introduced around the x-axis; (b) Statically determined system showing the Brazier pressure distribution and the virtual bending moments $M_{1,2}$ applied at the hinges (b).

curved beams was adopted where the radius of curvature is large in comparison with the cross-sectional dimensions as discussed by Parkus [17]. In this way Euler-Bernoulli theory is preserved and $\mathrm{d} M / \mathrm{d} s=Q$ holds where $\mathrm{d} s$ is the infinitesimal arc length and $Q$ denotes the shear force in the local crosssection beam CSYS. The principle of virtual work - as for instance described in Boresi et al. [18] - was consequently used to solve the statically indetermined system. By neglecting the in-plane shear deformation, the problem can be written as follows

$$
\left[\begin{array}{ll}
\delta_{11} & \delta_{12} \\
\delta_{12} & \delta_{22}
\end{array}\right] \cdot\left\{\begin{array}{l}
X_{1} \\
X_{2}
\end{array}\right\}=\left\{\begin{array}{l}
-\delta_{10} \\
-\delta_{20}
\end{array}\right\}
$$

with

$$
\delta_{i j}=\frac{1}{E I} \int_{0}^{\pi} M_{i} M_{j} R \mathrm{~d} \theta+\frac{1}{E A} \int_{0}^{\pi} N_{i} N_{j} R \mathrm{~d} \theta
$$

where indices 1 and 2 indicate section forces in the statically determined system due to virtually applied unit bending moments, and index 0 indicates section forces induced by the Brazier pressure $\mathrm{d} \vec{p}, M$ and $N$ indicate in-plane bending moments and normal forces respectively, $R$ denotes the radius of the circular cross section, $\theta$ represents the angular coordinate in a polar CSYS and $X_{1}$ and $X_{2}$ represent the unknown hinge moments in the statically indetermined system. The solution of equation 6 yields $X_{1}=X_{2}=\kappa^{2} E t R^{3} \mathrm{~d} z / 4$ which yields the moment distribution $M=$ $M_{0}+X_{1} M_{1}+X_{2} M_{2}=-\kappa^{2} E t R^{3} \mathrm{~d} z\left(2 \cos \theta^{2}-1\right) / 4$ and the axial force distribution as $N=N_{0}+X_{1} N_{1}+X_{2} N_{2}=\kappa^{2} E t R^{2} \mathrm{~d} z \cos \theta^{2}$. The in-plane strain 
energy $U_{1}$ for the entire section can hence be written as

$$
U_{1}=\int_{0}^{\pi}\left(\frac{M^{2}}{E I}+\frac{N^{2}}{E A}\right) R \mathrm{~d} \theta=\frac{3}{8} \frac{\kappa^{4} E R^{5} \pi\left(R^{2}+t^{2}\right) \mathrm{d} z}{t}
$$

The out-of-plane first order (St. Venant) strain energy $U_{0}$ can be written with $I_{x}=R^{3} t \pi$ as

$$
U_{0}=\frac{M_{x}^{2} \mathrm{~d} z}{2 E I_{x}}=\frac{\kappa^{2} E R^{3} t \pi \mathrm{d} z}{2}
$$

with equations 8 and 9 and with substitution of $\sigma=t / R$ (as performed in $[3]$ ), the total strain energy can consequently be written as follows

$$
U=U_{0}-U_{1}=\frac{E \pi R^{3} t \kappa^{2}}{2}\left(1-\frac{3}{4} \frac{R^{4} \kappa^{2}\left(1-\sigma^{2}\right)}{t^{2}}\right) \mathrm{d} z
$$

which is identical to equation 16 derived by Brazier [3] for initially straight tubes.

\section{Calculation}

\subsection{Analytical solution for asymmetrical, curved hollow sections}

In this section an analytical model of an asymmetrical, curved thin-walled mono-cellular cross section subjected to bi-axial bending is presented. Fig. 4 shows the adopted approach of subjecting a slice of the main beam to Brazier pressure. The geometry was chosen for the purpose of connecting two curved

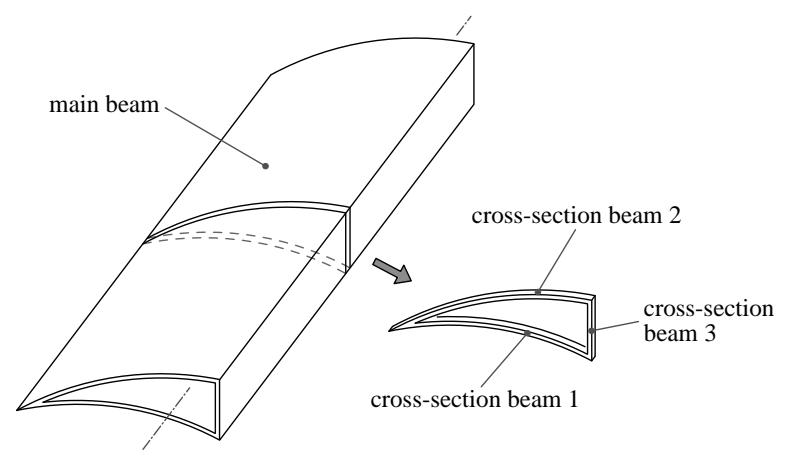

Figure 4: Slice with a width of $\mathrm{d} z$ taken from main beam and partitioned into three cross-section beams with a thickness of $t$.

cross-section beams at a shallow angle in such a way that they form a reentrant corner in point $A$. This detail is similar to the trailing edge of an 
airfoil (Fig. 1) which consequently will be the focus of this paper. Figure 5 (a) shows the geometry of the cross section which is defined by three key points $A, B, C$ that are located at the corners of an isosceles triangle - itself defined by the geometrical parameters $c=1.0 \mathrm{~m}$ and $h=0.3 \mathrm{~m}$.
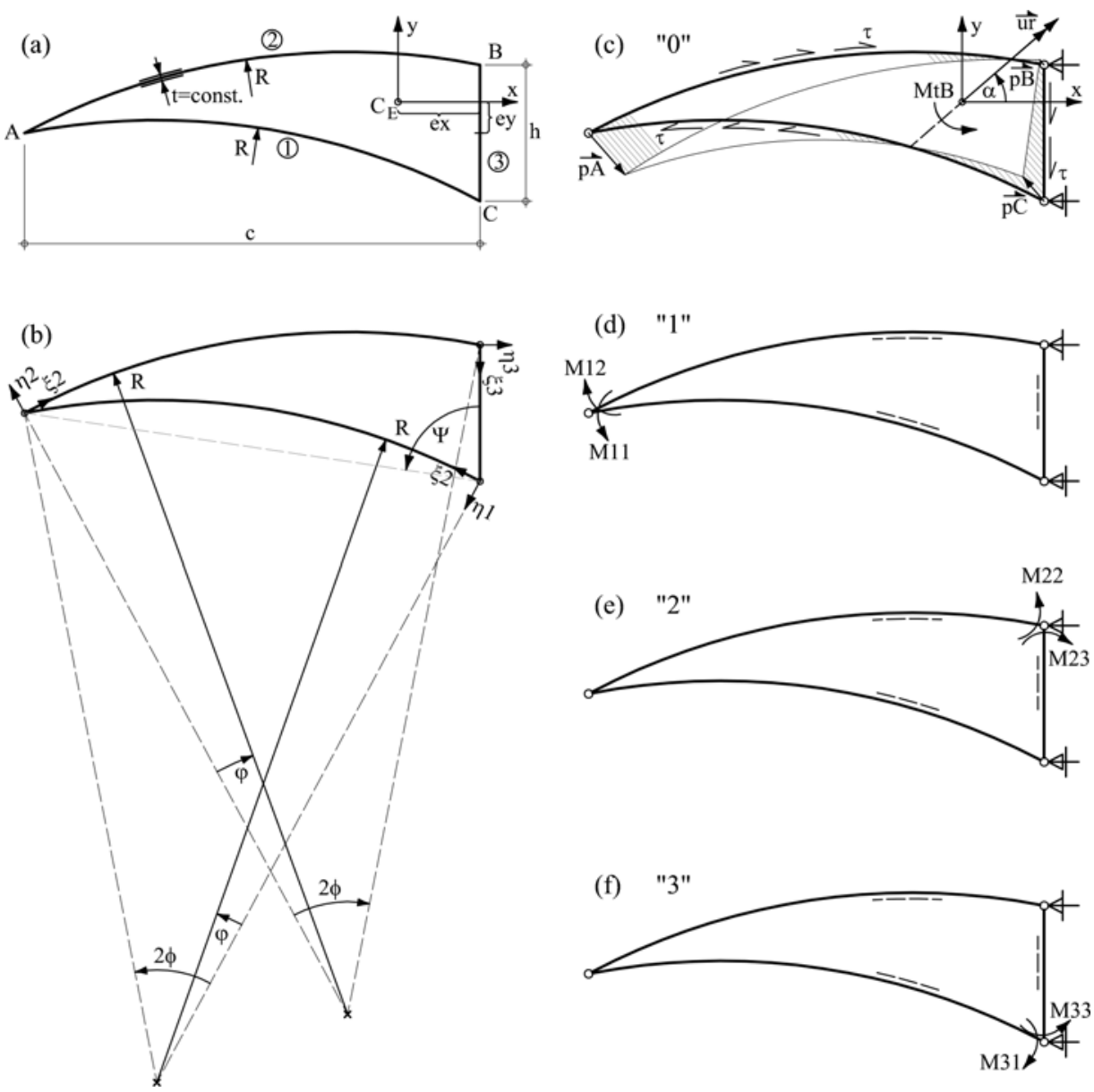

Figure 5: (a) Cross section with key points connected by curved cross-section beams and a straight vertical cross-section beam (web). Origin of CSYS given by $e_{x}$ and $e_{y}$ with reference to the mid-point of the web; (b) Definition of cross-section beam CSYS and parametrisation of cross section; (c) Rotation vector applied in $C_{E}$ with Brazier pressure distribution, Brazier torsion $M t_{B}$ and compensation shear stress $\tau$; (d) - (f) Virtual bending moments applied at hinges (indicated by circles) of statically determined system with boundary conditions. 
The key points $A, B$ and $A, C$ are connected by circular arches denoted as 1 and 2 with a radius $R$. The 3D shell finite element model presented in Subsection 3.2 was used to determine a wall thickness that prevents local buckling at the load levels investigated. The main beam section slice has a uniform wall thickness $t=0.01 \mathrm{~m}$ and a depth of $\mathrm{d} z=0.001 \mathrm{~m}$. The adopted material is High-Strength Low-Alloy (HSLA) steel with a yield strength of $f_{y}=8.0 \times 10^{8} \mathrm{~Pa}$, a fatigue threshold stress intensity factor of $\Delta K_{t h}=4.0 \times 10^{6} \mathrm{Pam}^{1 / 2}$ and a Young's modulus of $E=2.1 \times 10^{11} \mathrm{~Pa}$ (see e.g. Boyer $[19])$. Figure 5 (b) shows that a rotation vector $\overrightarrow{u_{r}}$ whose direction is given by $\alpha$ has been applied.

The position vectors $\vec{r}_{1,2,3}$ pointing from the origin $\left(C_{E}\right)$ to the axis of the particular cross-section beam can be parameterised as follows

$$
\begin{gathered}
\overrightarrow{r_{1}}=\left\{\begin{array}{c}
R(\cos (\psi-\phi+\varphi)-\cos (\psi-\phi))-e_{x} \\
R(\sin (\psi-\phi)-\sin (\psi-\phi+\varphi))-h / 2-e_{y}
\end{array}\right\} \\
\overrightarrow{r_{2}}=\left\{\begin{array}{c}
R(-\cos (\psi-\phi+\varphi)+\cos (\psi-\phi))-e_{x} \\
R(-\sin (\psi-\phi)-\sin (\psi-\phi+\varphi))+h / 2-e_{y}
\end{array}\right\} \\
\overrightarrow{r_{3}}=\left\{\begin{array}{c}
-e_{x} \\
y
\end{array}\right\}
\end{gathered}
$$

where $\psi$ denotes the angle between the secant and cross-section beam no. 3 , $\phi$ denotes the sector angle and $\varphi$ represents the integration angle; $e_{x}$ and $e_{y}$ represent geometrical distances that define the position of $C_{E}$ as stipulated in Fig.5 (a) and (b).

The Brazier pressure distribution acting on the cross-section beams in the global CSYS can generally be written as

$$
\vec{p}_{1,2,3}=\kappa^{2} \operatorname{Etd} z\left([T]^{-1}\left[\begin{array}{cc}
0 & 0 \\
0 & -1
\end{array}\right][T] \vec{r}_{1,2,3}\right)
$$

with $\vec{r}_{1,2,3}$ given by equations 11 through 13 and $T$ given by equation 2 .

Three degrees of freedom need to be constrained to prevent rigid body translations and rigid body rotation. The corresponding support reactions must be eliminated. Whilst the net Brazier pressures in the $\mathrm{x}, \mathrm{y}$-directions are necessarily zero, a Brazier torsion is induced into the cross section whose magnitude is given by equation 4. A constant shear stress (Fig.5 (c)) according to Bredt's first formula is applied in the opposite direction of $M t_{B}$ 
as follows

$$
\left|\vec{\tau}_{1,2,3}\right|=M t_{B} /\left(2 A_{0} t\right)
$$

where $A_{0}$ represents the area enclosed by the centre lines of the cross-section beams.

The system was made statically determined by introducing three rotational hinges at the key points. Pairs of virtual bending moments were applied at the cross-section beam ends in points $A, B$ and $C$ as indicated in Fig.5 (d)-(f). The required global section force vector at the key points in cross-section beams 1 and 2 can be obtained with

$\vec{F}=\left\{F x_{1 A}, F y_{1 A}, F x_{2 A}, F y_{2 A}, F x_{2 B}, F y_{2 B}, F x_{1 C}, F y_{1 C}\right\}^{T}=P^{-1} \cdot L$ where $x$ and $y$ indicate the nodal force direction, indices 1 and 2 indicate the crosssection beam number, $P$ represents a matrix that contains the global key point coordinates and $L$ represents a load vector, both of which are defined in the Appendix. The axial forces $N_{1,2,3}$ and shear forces $Q_{1,2,3}$ in the crosssection beams are obtained by transformation of the corresponding components of $\vec{F}$ into the $\xi, \eta$ cross-section beam CSYS. The section moments can be separately obtained by $M_{1,2}=\int_{0}^{2 \phi} Q_{1,2} R \mathrm{~d} \varphi$ and $M_{3}=\int_{0}^{h} Q_{3} \mathrm{~d} \xi$.

Consequently, the following system of linear equations can be used to solve for the unknown corner-bending moments $M_{A}, M_{B}$ and $M_{C}$ as follows

$$
\left[\begin{array}{lll}
\delta_{11} & \delta_{12} & \delta_{13} \\
\delta_{21} & \delta_{22} & \delta_{23} \\
\delta_{31} & \delta_{32} & \delta_{33}
\end{array}\right] \cdot\left\{\begin{array}{l}
M_{A} \\
M_{B} \\
M_{C}
\end{array}\right\}=\left\{\begin{array}{l}
-\delta_{10} \\
-\delta_{20} \\
-\delta_{30}
\end{array}\right\}
$$

with

$$
\begin{aligned}
\delta_{i j} & =\frac{1}{E I}\left[\int_{0}^{2 \phi} M_{i 1} M_{j 1} R \mathrm{~d} \varphi+\int_{0}^{2 \phi} M_{i 2} M_{j 2} R \mathrm{~d} \varphi+\int_{0}^{h} M_{i 3} M_{j 3} \mathrm{~d} \xi\right] \\
& +\frac{1}{E A}\left[\int_{0}^{2 \phi} N_{i 1} N_{j 1} R \mathrm{~d} \varphi+\int_{0}^{2 \phi} N_{i 2} N_{j 2} R \mathrm{~d} \varphi+\int_{0}^{h} N_{i 3} N_{j 3} \mathrm{~d} \xi\right]
\end{aligned}
$$

where indices $i, j$ running from 1-3 represent the number of the system with virtual section forces, and 0 represents the system subject to Brazier pressure and compensating shear stress.

The in-plane bending moment induced by the Brazier pressure in corner point $A$ can be written as follows

$$
M_{A}=-\kappa^{2} E \mathrm{~d} z\left(c_{1} \sin \alpha \cos \alpha+c_{2} \cos \alpha^{2}+c_{3}\right)
$$


where the geometrical constants $c_{1}, c_{2}$ and $c_{3}$ are second-order polynomial functions of the cross-section beam curvature $1 / R$.

Note that the expressions for these constants are lengthy and therefore not reproduced here for the sake of brevity. Also note the similarity between equations 18 and 4 . The angle $\alpha_{\max }$ for the maximum opening mode bending moments $\mathrm{d} M_{A} / \mathrm{d} \alpha=0$ can be written as follows:

$$
\alpha_{\max }=\frac{n \pi}{2}+\arctan \left(\frac{-c_{2}+\sqrt{c_{1}^{2}+c_{2}^{2}}}{c_{1}}\right)
$$

where $n=1,3, \ldots$ and the constants as stipulated in equation 18 .

The corner moment $M_{A}$ usually causes in-plane bending stresses that are considerably lower than those caused by out-of-plane bending. However, the importance of $M_{A}$ manifests itself in the susceptibility of the joint to fatigue damage. Therefore, an analytical double cantilever beam (DCB) model was used to estimate the stress intensity factor $\Delta K_{A}$ in a small crack that is considered to be already present in the re-entrant corner. Such a crack could be represented by a flaw that internally runs along the trailing edge. Figure 6 shows the DCB model where the analytical expression for the energy release rate $G$ given in Sørensen et al. [20] was used to compute $\Delta K_{A}$ as follows

$$
\Delta K_{A}=\sqrt{G E}=\sqrt{12 M_{A}^{2} /\left(\mathrm{d} z^{2} t^{3}\right)}
$$
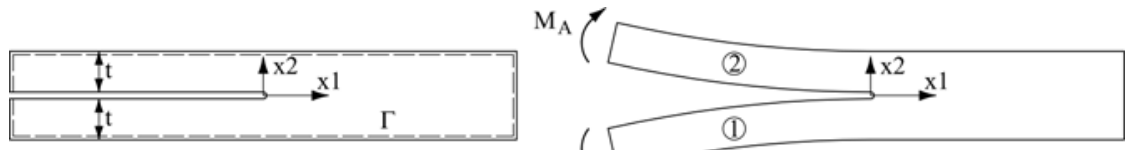

(a)

(b)

Figure 6: Linear elastic isotropic double cantilever beam (DCB) model used to obtain $\Delta K_{A}$ for a pure Mode-I loading condition where the contribution of axial and shear forces in the beams was disregarded. The DCB represents a simplification of the actual joint geometry but is still considered to be a reasonable approximation when estimating the stress intensity factor. Note that $\Delta K_{A}$ of the DCB loaded in pure bending is independent of the crack length.

\subsection{Numerical analysis}

For the numerical analysis the commercial finite element package ABAQUS [21] was used. In this study a 3D model of the problem is used to corroborate 
the analytically obtained forces and in-plane displacements. The geometrical and material properties of the cross section are described in Subsection 3.1; the remaining parameters are given in Tab. 1. The cross section was uni-

Table 1: Dimensions and analytically computed section properties for the cross section as defined in Fig.5 (a) and used for the numerical model.

\begin{tabular}{ccccccc}
\hline$R$ & $e_{x}$ & $e_{y}$ & $A_{0}$ & $I_{x}$ & $I_{y}$ & $I_{x y}$ \\
{$[\mathrm{~m}]$} & {$[\mathrm{m}]$} & {$[\mathrm{m}]$} & {$\left[\mathrm{m}^{2}\right]$} & {$\left[\mathrm{m}^{4}\right]$} & {$\left[\mathrm{m}^{4}\right]$} & {$\left[\mathrm{m}^{4}\right]$} \\
\hline 2.0 & -0.436 & 0.037 & 0.15 & $1.885 \times 10^{-4}$ & $2.374 \times 10^{-3}$ & $-6.580 \times 10^{-5}$ \\
\hline
\end{tabular}

formly extruded (i.e. without taper and twist) in the positive $z$-direction to a total main beam length of $20 \mathrm{~m}$. The right-handed global CSYS corresponds to the one shown in Fig.5 (a). The main beam model was discretized with $3.34 \times 10^{4}$ four noded quadrilateral shell elements with reduced integration and hourglass control (Abaqus type S4R). The cross section was discretized with 120 elements in the circumferential direction without offset. Rigid (kinematic) coupling constraints were assigned to both ends. The master nodes of the constraints were located in the elastic centres of the end sections. Only the in-plane displacements $u_{x}$ and $u_{y}$ were decoupled in order to allow for in-plane deformation. Table 2 gives the displacement boundary conditions that were assigned to the numerical model. Diametrically opposed rotations

Table 2: Six essential boundary conditions assigned to the main beam mid-section at $z=$ $10.0 \mathrm{~m}$ in points $A, B$ and $C$. Essential boundary conditions of the master nodes at $z=$ $0 \mathrm{~m}$ and $z=20.0 \mathrm{~m}$ respectively.

\begin{tabular}{cccc}
\hline Node & $u_{x}$ & $u_{y}$ & $u_{z}$ \\
\hline $\mathrm{A}$ & fixed & fixed & fixed \\
$\mathrm{B}$ & fixed & free & fixed \\
$\mathrm{C}$ & free & free & fixed \\
Master & fixed & fixed & free \\
\hline
\end{tabular}

$u r_{x}$ and $u r_{y}$ were simultaneously applied to the master nodes in several increments where the following relation between curvature and cross-sectional rotation holds for small rotations $\kappa=|\overrightarrow{u r}| / \mathrm{d} z$. A static implicit solver technique was used where geometric nonlinearity on the basis of the GreenLagrange formulation was used. The Brazier torsion of the numerical model was computed with $M t_{B}=2 \tau_{m} A_{0} t$ where $\tau_{m}$ was obtained by integration of the shear stress according to $\tau_{m}=1 / l \int_{0}^{l} \tau_{(s)} \mathrm{d} s ; l$ denotes the circumference of the cross section and $\tau_{(s)}$ the numerically obtained nodal shear stress. 


\section{Results}

\subsection{Analytical results}

In order to facilitate the comparison of the numerical results with the analytical solution, the analytical constants of equation 18 are given in Tab. 3 for the present example. Figure 7 (a) shows a contour plot of $M_{A} /\left(\kappa^{2} E \mathrm{~d} z\right)$

Table 3: Constants as functions of the geometrical parameters $c, h, t$ and $R$ for various cross-section beam curvatures $1 / R$ where $\alpha_{\max }$ is obtained from equation 19 for $n=1$.

\begin{tabular}{ccccc}
\hline $\begin{array}{c}1 / R \\
{[1 / \mathrm{m}]}\end{array}$ & $\begin{array}{c}c_{1} \\
{\left[\mathrm{~m}^{4}\right]}\end{array}$ & $\begin{array}{c}c_{2} \\
{\left[\mathrm{~m}^{4}\right]}\end{array}$ & $\begin{array}{c}c_{3} \\
{\left[\mathrm{~m}^{4}\right]}\end{array}$ & $\begin{array}{c}\alpha_{\max } \\
{\left[{ }^{\circ}\right]}\end{array}$ \\
\hline 0.0 & $4.1530 \times 10^{-7}$ & $5.0802 \times 10^{-5}$ & $1.3882 \times 10^{-5}$ & 90 \\
0.1 & $4.6670 \times 10^{-5}$ & $5.0150 \times 10^{-5}$ & $1.4367 \times 10^{-5}$ & 111 \\
0.2 & $9.3033 \times 10^{-5}$ & $4.8202 \times 10^{-5}$ & $1.5818 \times 10^{-5}$ & 121 \\
0.3 & $1.3893 \times 10^{-4}$ & $4.4973 \times 10^{-5}$ & $1.8224 \times 10^{-5}$ & 126 \\
0.4 & $1.8368 \times 10^{-4}$ & $4.0538 \times 10^{-5}$ & $2.1533 \times 10^{-5}$ & 129 \\
0.5 & $2.2746 \times 10^{-4}$ & $3.4920 \times 10^{-5}$ & $2.5730 \times 10^{-5}$ & 131 \\
0.6 & $2.6987 \times 10^{-4}$ & $2.8208 \times 10^{-5}$ & $3.0753 \times 10^{-5}$ & 132 \\
0.7 & $3.1092 \times 10^{-4}$ & $2.0461 \times 10^{-5}$ & $3.6567 \times 10^{-5}$ & 133 \\
\hline
\end{tabular}

as a function of the cross-section beam curvature $1 / R$ and the loading angle. Figure 7 (b) shows sections taken parallel to the ordinate for three different cross-section beam curvatures. It can be seen that $M_{A}$ is a periodic function of $\alpha$ that forms ridges whose magnitude increases with increasing cross-section beam curvature. In the limit case of $1 / R=0$ (i.e. straight cross-section beams) only negative moments $M_{A}$ can be obtained, which corresponds to a closing for any loading angle. Conversely, the section at $1 / R$ $=0.7 \mathrm{~m}^{-1}$ shows the presence of positive opening moments whose peaks occur at about $\alpha=135^{\circ}$ and $\alpha=315^{\circ}$. Figure 8 (a) shows a contour plot of $\Delta K_{A} / \Delta K_{t h}$ for elastic bending curvatures $\kappa_{e l}=f_{y} /(E r)$ which satisfy the condition that the absolute value of the highest stress point in the cross section is equal to $f_{y}$. Most importantly, Fig. 8 (b) shows that a critical cross-section beam curvature $1 / R_{\text {crit }}$ exists at which $\Delta K_{A}$ reaches the fatigue threshold. 


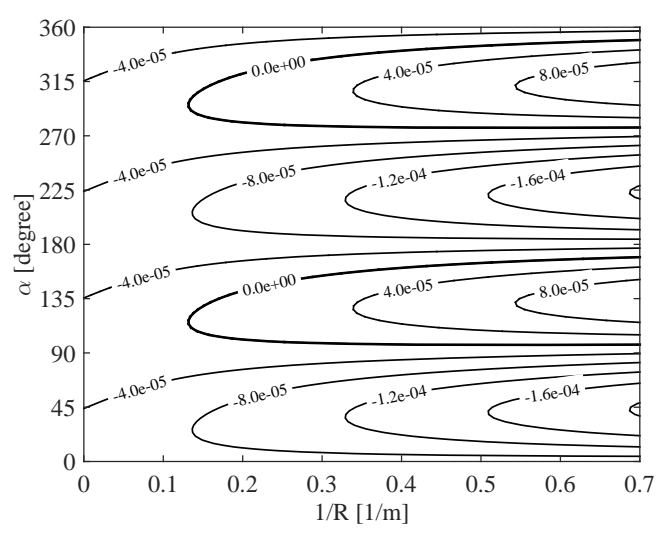

(a) $M_{A} /\left(\kappa^{2} E \mathrm{~d} z\right)$

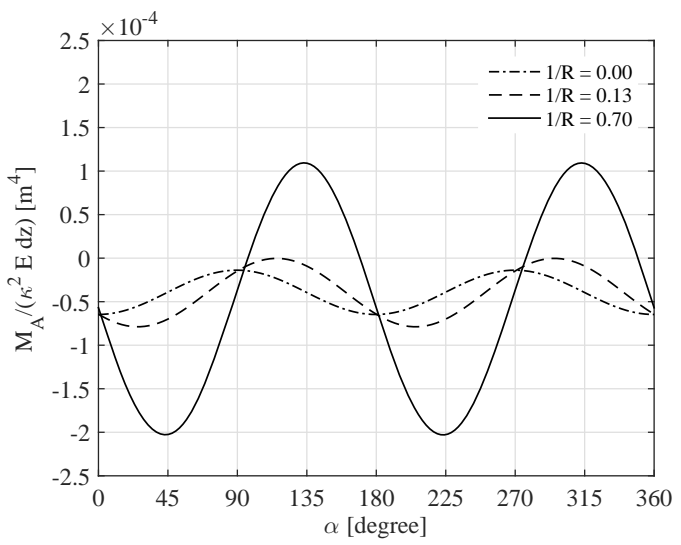

(b)

Figure 7: (a) Positive contours refer to an opening mode of the corner. Zero contours are indicated in bold solid lines; (b) Section of 3D function $M_{A} /\left(\kappa^{2} E \mathrm{~d} z\right)$. Dash-dotted graph represents straight cross-section beams where $\max \left(M_{A}\right)<0$ for any arbitrary $\alpha$. Dashed graph represents $\max \left(M_{A}\right)=0$ and the solid line represents $\max \left(M_{A}\right)>0$.

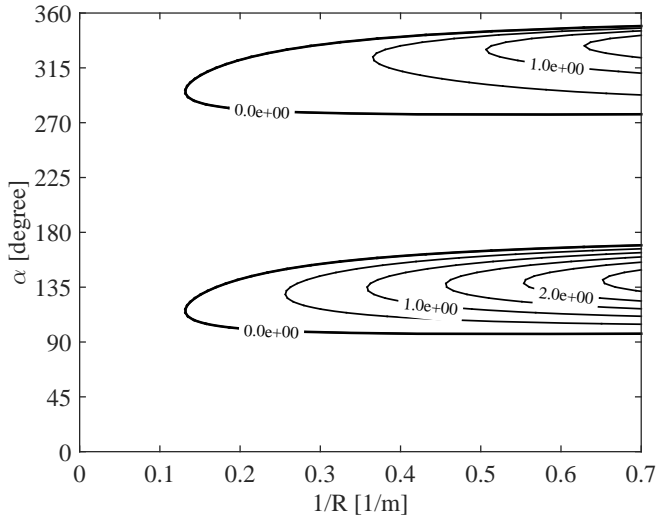

(a) $\Delta K_{A} / \Delta K_{t h}$

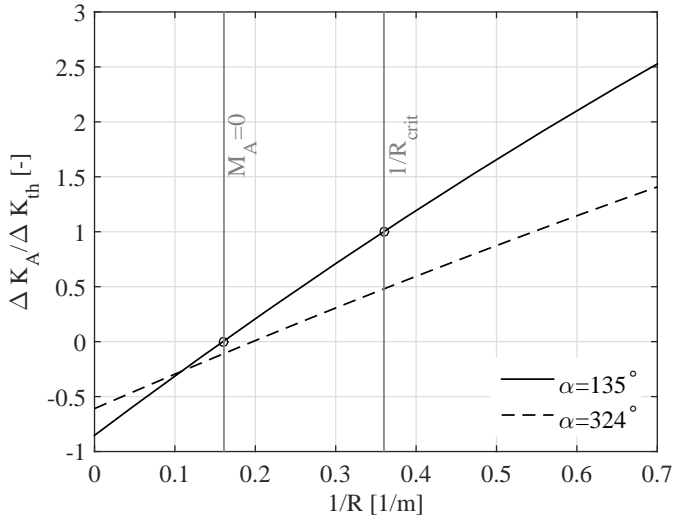

(b)

Figure 8: (a) Contour plot of normalised $\Delta K_{A} / \Delta K_{t h}$ for $M_{A} \geq 0$ with two ridges at $\alpha$ $=135^{\circ}$ and $\alpha=324^{\circ}$; (b) Sections taken parallel to the abscissa showing $\Delta K_{A} / \Delta K_{t h}$ as second-order function of the cross-section beam curvature $1 / R$ where the line $M_{A}=0$ indicates the limit curvature at which the cross-section beams start to 'open'.

Figure 9 (a) shows the normalised axial force in node $A$ for cross-section beams 1 and 2 for a cross-section beam curvature $1 / R=0.7 \mathrm{~m}^{-1}$ as a function of $\alpha$. Comparison of Fig. 7 (b) with Fig. 9 (a) shows that the peaks occur at 


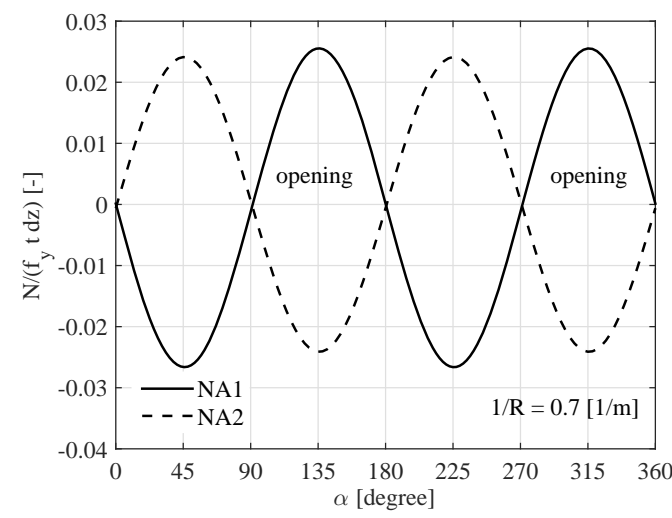

(a)

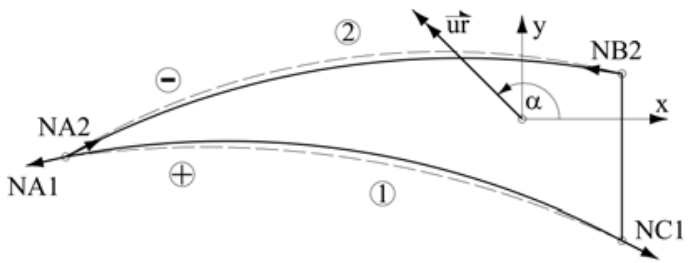

(b)

Figure 9: (a) Axial force in both cross-section beams in node A for $\kappa=$ const. normalised with elastic section capacity $f_{y} t \mathrm{~d} z$. Shows that positive axial force in lower cross-section beam $N_{A 1}$ and negative axial force in upper cross-section beam $N_{A 2}$ corresponds to a positive (opening) bending moment $M_{A}$. The angles of the bending axis $\alpha=135^{\circ}$ and $\alpha=315^{\circ}$ with maximum positive $M_{A}$ correspond well with $\max \left(N_{A 1}\right)$ and $\min \left(N_{A 2}\right)$ respectively; (b) Illustration of axial force components in the curved cross-section beams with tension in lower cross-section beam and compression in upper cross-section beam for loading angle of $\alpha=135^{\circ}$.

the same loading angles where a positive $M_{A}$ is accompanied by tension in cross-section beam 1 and compression in cross-section beam 2. Figure 9 (b) shows that owing to eccentricity moments the lower cross-section beam tends to decrease its curvature and the upper cross-section beam to increase its curvature when subjected to tension and compression respectively. Although the magnitude of the axial force is rarely affected by the cross-section beam curvature, its influence is primarily caused by moments of eccentricity that counteract the bending moments directly induced by the Brazier pressure.

Figure 10 (a) and (b) show the in-plane deformations of a cross section with curved and straight cross-section beams for two different loading angles. It can be seen that the angle of the applied rotation vector $\alpha=0$ leads to a closing of corner $A$ in both cases. Conversely, the corner opens for a curved cross-section beam which exceeds a certain radius (indicated by $M_{A}=0$ in Fig. 8) and closes for the triangular cross section for $\alpha=135^{\circ}$.

\subsection{Numerical results}

Figure 11 (a) shows a superimposed plot of the undeformed and deformed shape of the finite element shell model whereas Fig. 11 (b) shows a super 


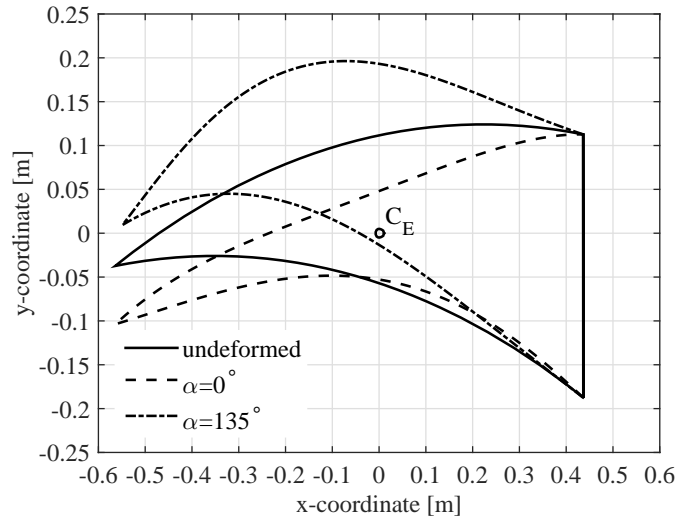

(a)

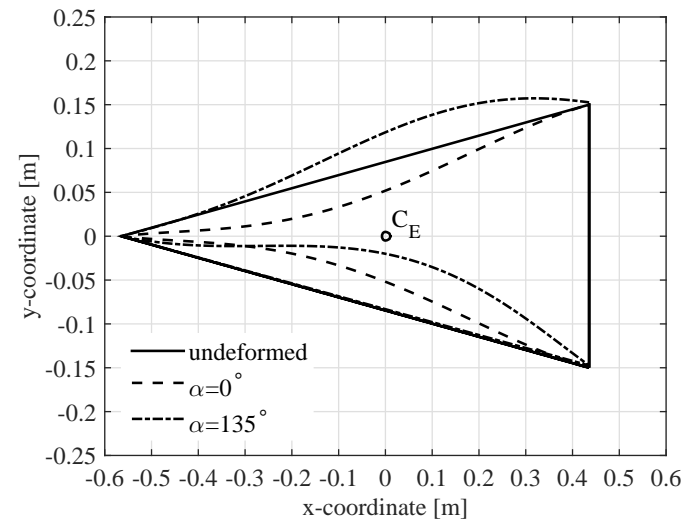

(b)

Figure 10: (a) Cross section with cross-section beam radius $R=2 \mathrm{~m}$ subjected to $\kappa=$ $0.01 \mathrm{~m}^{-1}$. Dashed graph shows in-plane deformation for $\alpha=0^{\circ}$ and dash-dotted graph for $\alpha=135^{\circ}$ respectively. It is interesting to see that the trailing edge curls in and out depending on the bending direction; (b) Cross section limit case for $R=\infty$. No curling effect for straight cross-section beam independently of loading direction; Deformations of both figures are scaled by a factor of 10 where the solid line shows the undeformed cross section.

imposed cross-sectional deformation plot of the mid-slice of the same model. Figure 12 (a) compares the analytically (see equation 4) and numerically

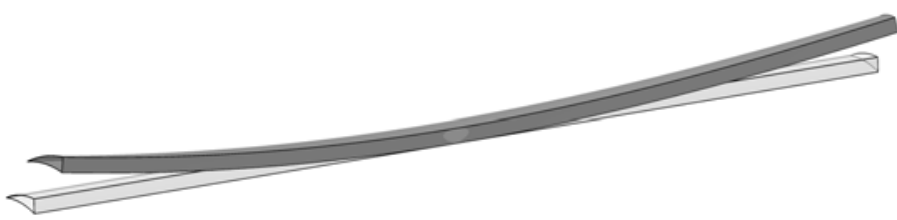

(a)

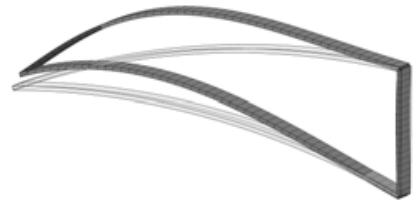

(b)

Figure 11: (a) Global deformation of the main beam model for a loading angle of $\alpha=$ $135^{\circ}$ at $\kappa=0.01 \mathrm{~m}^{-1}$; (b) in-plane deformation at mid-span for the same load case with a deformation scaling factor of 10 .

obtained Brazier torsion gradient $M t_{B} / \mathrm{d} z$ for $\alpha=0^{\circ}$ and $\alpha=135^{\circ}$ evaluated at the mid-span. The main beam would have reached the elastic torsional capacity $M t_{e l}=2 \tau_{\text {oct }} A_{0} t=2 \sqrt{2} f_{y} A_{0} t / 3$ at a total main beam length of approx. $88 \mathrm{~m}$ for a curvature of $\kappa=0.01 \mathrm{~m}^{-1}$. Figure 12 (b) compares the inplane bending moments obtained from equation 18 with the corresponding 


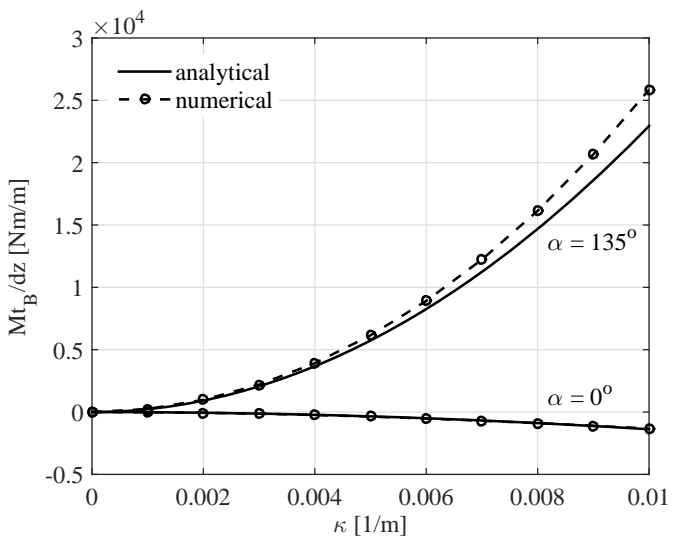

(a)

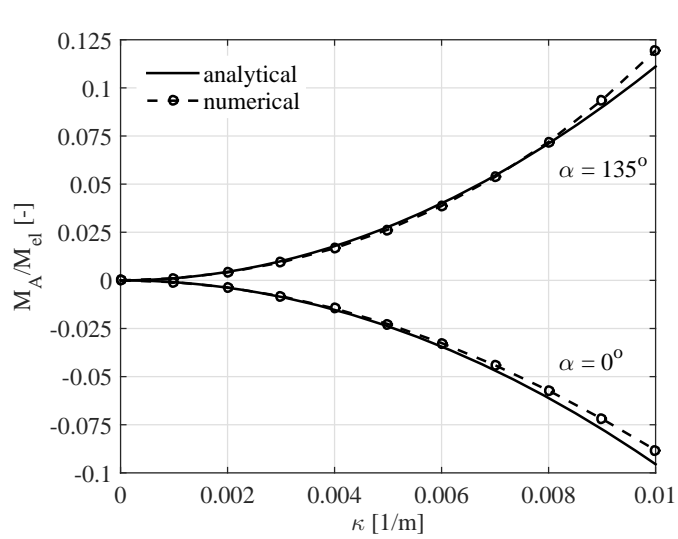

(b)

Figure 12: (a) Brazier torsion gradient $M t_{B} / \mathrm{d} z$ as a function of the main beam bending curvature $\kappa$; (b) Normalised in-plane bending moment $M_{A} / M_{e l}$ (b) per unit length.

numerical results at mid-span for the same loading directions. Both $M t_{B}$ and $M_{A}$ are quadratic functions of the bending curvature $\kappa$. Furthermore, it can be seen that $M_{A}$ induces in-plane stress levels in the cross-section beams that are well below the elastic bending moment capacity of the cross-section beams $M_{e l}=f_{y} t^{2} / 6$ per unit length.

Table 4 lists the analytically and numerically obtained in-plane bending moments along with the relative error for three different loading directions. Figure 13 (a) and (b) compare the analytical in-plane deformation with the

Table 4: Comparison of normalised $M_{A} / M_{e l}$ obtained from analytical expression and numerical analysis for a constant curvature of $\kappa=0.01 \mathrm{~m}^{-1}$

\begin{tabular}{cccc}
\hline Model & $\alpha=0^{\circ}$ & $\alpha=45^{\circ}$ & $\alpha=135^{\circ}$ \\
\hline Analytical & $-9.550 \times 10^{-2}$ & $-2.178 \times 10^{-1}$ & $1.111 \times 10^{-1}$ \\
Numerical & $-8.819 \times 10^{-2}$ & $-2.371 \times 10^{-1}$ & $1.194 \times 10^{-1}$ \\
Rel.error & $7.65 \%$ & $8.84 \%$ & $7.03 \%$ \\
\hline
\end{tabular}

shell model deformations at mid-span. It can be seen that the analytical second-order model overestimates the numerically obtained deformations in both cases. 


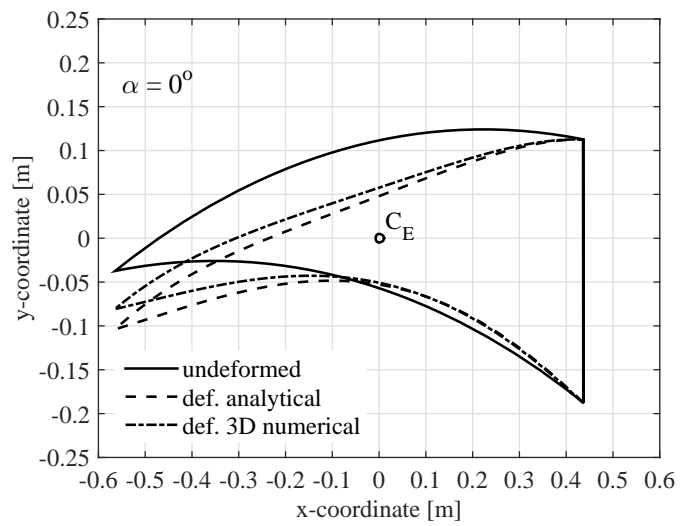

(a)

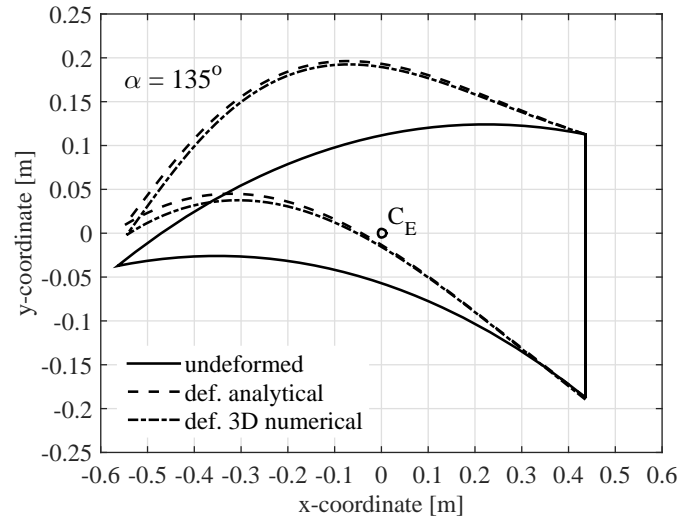

(b)

Figure 13: In-plane cross section deformations between analytical second order 2D and numerical fully nonlinear 3D shell model for $R=2 \mathrm{~m}$ for $\alpha=0^{\circ}$ and for $\alpha=135^{\circ}$. Deformation scaling factor 10 .

\section{Discussion}

It can be shown analytically that slender, thin-walled, initially straight linear elastic isotropic main beams exhibit a second-order coupling between bending and twisting. This coupling effect is related to the misalignment between the rotation vector and the bending moment vector. It is evident that the analytically obtained Brazier torsion agrees well with the numerically computed torsional moment in the cross section of a 3D shell model. The numerical simulation was necessarily fully nonlinear whereas the analytical solution is based on second-order theory. That is to say, that the analytical Brazier pressure is based on the undeformed geometry of the cross section, which explains the increasing deviation with increasing $\kappa$ in Fig.12 (a) and (b).

Interestingly the analytical results presented in Fig. 7 are consistent with the measurements of local relative displacements taken in vicinity of the trailing edge of a SSP34 wind turbine blade. Figure 14 shows two different combined edgewise-flapwise loading directions and the associated bending moment vectors as conducted in two separate blade tests reported in [22] and [13]. The trailing edge was closing for a resulting bending moment direction of $\alpha=210^{\circ}$ and opening for a bending moment direction of $\alpha=150^{\circ}$. 
From both, the analytical as well as experimental results, it could be grossly inferred that bending axes or bending moments within the first and the third quadrant cause a closing whereas bending axes within the second and the fourth quadrant cause an opening effect. It could be shown analytically

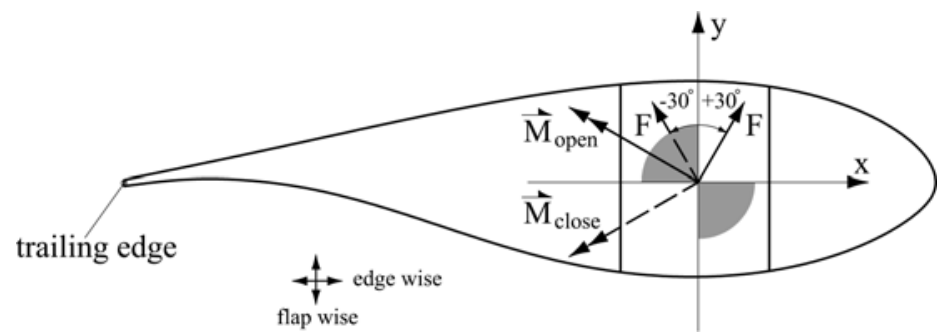

Figure 14: Schematic airfoil with two combined experimental edgewise-flapwise loading directions under $+30^{\circ}$ and $-30^{\circ}$ with corresponding moment vectors $\vec{M}_{\text {open }}$ and $M_{\text {close }}$. Moment vectors within the grey hatched quadrants are potentially leading to opening modes of the trailing edge joint.

that the counterintuitive opening effect is caused by the axial forces acting in conjunction with the curvature of the cross-section beams. Due to eccentricity moment effects the curved cross-section beams start to diverge from one another at certain loading angles; this effect vanishes in the case of straight cross-section beams. Although the in-plane stresses in the crosssection beams induced by the Brazier effect are small, the opening deformation mode is considered to be a significant fatigue driver in re-entrant corners under high-cycle loading conditions. Comparison of $\Delta K_{A} / \Delta K_{t h}$ shows that depending on the cross-section beam radius, the fatigue threshold can easily be exceeded by a factor of 2.5 in the example presented. It is deemed that the opening effect is even amplified in main beams with orthotropic material behaviour which exhibit a low in-plane bending stiffness when compared to their out-of-plane stiffness.

\section{Conclusions}

It can be concluded that geometric nonlinear effects are important for fatigue analysis of wind turbine blades at bending moment levels that are lower than the Brazier limit and presumably lower than the local stability limit. More precisely the curvature of the trailing edge panels (i.e. camber) of airfoils has a considerable influence on the fatigue behaviour of trailing edge joints. 
The suggested approach serves as a basis for computationally efficient fracture analysis of geometrically nonlinear effects in realistic wind turbine blade cross sections.

\section{Acknowledgements}

The work is supported by the Danish Energy Agency through the 2010 Energy Technology Development and Demonstration Program (EUDP). The supported EUDP-project titled is "Experimental Blade Research - Phase 2" and has journal no. 64011-0006. The support is gratefully acknowledged. The first author is grateful for the discussions with Alexander Stäblein from DTU Wind Energy which inspired the analytical work.

\section{References}

[1] T. von Kármán, Über die Formänderung dünnwandiger Rohre, insbesondere federnder Ausgleichrohre, Z. d. Vereines deutscher Ingenieure 45 (1911) 1889-1895.

[2] S. Timoshenko, Bending stresses in curved tubes of rectangular crosssection, Transactions of the American Society of Mechanical Engineers 45 (1923) 135-140.

[3] L. G. Brazier, On the Flexure of Thin Cylindrical Shells and other 'Thin' Sections, Proc. R. Soc. Lond. Ser. A, 116 (1927) 104-114.

[4] R. A. Clark, E. Reissner, Bending of curved tubes, Adv. Appl. Mech. 2 (1951) 93-122.

[5] E. Reissner, On Finite Bending of Pressurized Tubes, Transactions of the ASME (1959) 386-392.

[6] F. Guarracino, On the analysis of cylindrical tubes under flexure: theoretical formulations, experimental data and finite element analysis, Thin-Walled Structures 41 (2003) 127-147.

[7] E. Corona, R. A., Bending of Long Cross-Ply Composite Circular Cylinders, Composites Engineering 5 (2) (1995) 163-182. 
[8] F. Guarracino, A Simple Formula for Complementing FE Analyses in the Estimation of the Effects of Local Conditions in Circular Cylindrical Shells, CMES 72 (3) (2011) 167-184.

[9] F. Guarracino, M. Fraldi, A. Giordano, Analysis of testing methods of pipelines for limit state design, Applied Ocean Research 30 (2008) 297304.

[10] F. Guarracino, A. Walker, A. Giordano, Effects of Boundary Conditions on Testing of Pipes and Finite Element Modelling, Int. J. Press. Ves. Piping 86 (2009) 196-206.

[11] L. Kühlmeier, Buckling of wind turbine rotor blades, Ph.D. thesis, Aalborg University (2007).

[12] L. Damkilde, B. Lund, A Simplified Analysis of the Brazier Effect in Composite Beams, Civil-Comp Press (207) (2009) 1-11.

[13] J. Shmueli, M. A. Eder, A. Tesauro, A versatile stereo photogrammetry based technique for measuring fracture mode displacements in structures, Precision Engineering Article in Press (2014) 9.

[14] L. S. Cecchini, P. M. Weaver, Brazier Effect in Multibay Airfoil Sections, AIAA Journal 43 (10) (2005) 2252-2258.

[15] E. N. Jacobs, K. E. Ward, R. M. Pinkerton, The characteristics of 78 related airfoil sections from tests in the variable-density wind tunnel, Tech. rep., NACA report No. 460 (1933).

[16] Toronto: Maplesoft, a division of Waterloo Maple Inc., MAPLE, Algebraic Manipulations Software Package, ver 18 (2014).

[17] H. Parkus, Mechanik der festen Körper, Springer Wien NewYork, 1966.

[18] A. P. Boresi, K. Chong, J. D. Lee, Elasticity in Engineering Mechanics, John Wiley \& Sons, 2010.

[19] H. E. Boyer, Atlas of Fatigue Curves, Technology \& Engineering, 1985.

[20] B. F. Sørensen, K. Jørgensen, T. K. Jacobsen, R. C. Østergaard, DCBspecimen loaded with uneven bending moments, International J Fract 141 (2006) 163-176. 
[21] Dassault Systèmes, Abaqus Analysis User's Manual (2011).

[22] A. Tesauro, M. A. Eder, M. Nielsen, Measurement of local relative displacementsin large structures, J Strain Anal Eng 49 (2014) 301-314.

\section{Appendix}

The entities in matrix $P$ can be obtained from equations 11 and 12 by substitution of $\varphi=0$ and $\varphi=2 \phi$ respectively.

$$
P=\left[\begin{array}{cccccccc}
A_{y} & -A_{x} & 0 & 0 & 0 & 0 & C_{y} & C_{x} \\
0 & 0 & A_{y} & -A_{x} & B_{y} & -B_{x} & 0 & 0 \\
0 & 0 & 0 & 0 & B_{y} & -B_{x} & C_{y} & C_{x} \\
1 & 0 & 0 & 0 & 0 & 0 & 1 & 0 \\
0 & 1 & 0 & 0 & 0 & 0 & 0 & 1 \\
0 & 0 & 1 & 0 & 1 & 0 & 0 & 0 \\
0 & 0 & 0 & 1 & 0 & 1 & 0 & 0 \\
0 & 0 & 0 & 0 & 1 & 0 & 1 & 0
\end{array}\right]
$$

The load vectors for the statically determined systems labelled as $0,1,2$ and 3 in Fig. 5 (c)-(f) can be written as follows

$$
\begin{aligned}
& L_{0}=\left\{-M t_{1},-M t_{2},-M t_{1}-M t_{2},-G_{x 1},-G_{y 1},-G_{x 2},-G_{y 2},-G_{x 1}-G_{x 2}\right\}^{T} \\
& L_{1}=\left\{M_{11}, M_{12}, 0,0,0,0,0,0\right\}^{T} \\
& L_{2}=\left\{0, M_{22}, M_{23}, 0,0,0,0,0\right\}^{T} \\
& L_{3}=\left\{M_{31}, 0, M_{33}, 0,0,0,0,0\right\}^{T}
\end{aligned}
$$

where $M t_{1,2}$ are the Brazier torsion contributions of cross-section beams 1 and 2 which can be obtained as follows:

$$
M t_{1,2}=\int_{0}^{2 \phi}\left\{\vec{p}_{1,2}+\vec{\Gamma}_{1,2}\right\} \times \vec{r}_{1,2} R \mathrm{~d} \varphi
$$

and $G_{x 1,2}$ and $G_{y 1,2}$ are the total force contributions of cross-section beams 1 and 2 in the $x, y$ directions which can be written as follows:

$$
G_{x 1,2}=\int_{0}^{2 \phi} \vec{p}_{x 1,2}+\vec{\Gamma}_{x 1,2} R \mathrm{~d} \varphi
$$




$$
G_{y 1,2}=\int_{0}^{2 \phi} \vec{p}_{y 1,2}+\vec{\Gamma}_{y 1,2} R \mathrm{~d} \varphi
$$

where $\vec{p}_{1,2}$ is the Brazier pressure vector given by equation $14, \vec{r}_{1,2}$ is the position vector defined in equations 11 through 13 and $\vec{\Gamma}_{1,2}$ represents the compensation shear flow vector in global coordinates which is defined as $\left|\vec{\Gamma}_{1,2}\right|=\left|\vec{\tau}_{1,2}\right| t$ using equation 15 . 